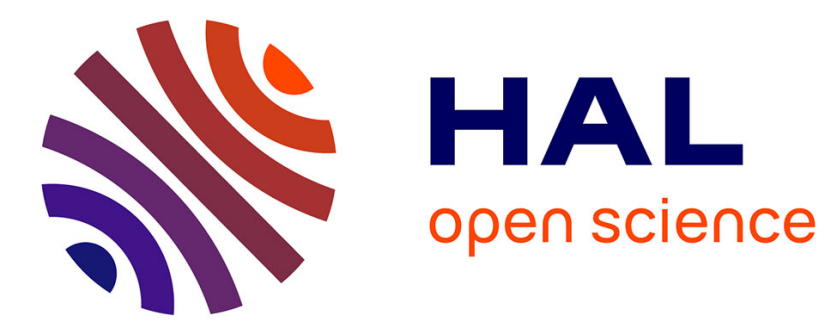

\title{
Consistent conjectures in a dynamic model of non-renewable resource management
}

Nicolas Querou, Mabel Tidball

\section{To cite this version:}

Nicolas Querou, Mabel Tidball. Consistent conjectures in a dynamic model of non-renewable resource management. 19. Annual Conference EAERE, European Association of Environmental and Resource Economists (EAERE). Venise, INT., Jun 2012, Prague, France. 29 p. hal-01402597

\section{HAL Id: hal-01402597 https://hal.science/hal-01402597}

Submitted on 24 Nov 2016

HAL is a multi-disciplinary open access archive for the deposit and dissemination of scientific research documents, whether they are published or not. The documents may come from teaching and research institutions in France or abroad, or from public or private research centers.
L'archive ouverte pluridisciplinaire HAL, est destinée au dépôt et à la diffusion de documents scientifiques de niveau recherche, publiés ou non, émanant des établissements d'enseignement et de recherche français ou étrangers, des laboratoires publics ou privés. 


\title{
Consistent conjectures in a dynamic model of non-renewable resource management
}

\author{
Nicolas Querou et Mabel Tidball
}

\begin{abstract}
We consider a model of non-renewable resource extraction where players do not know their opponents' utility functions and form conjectures on the behavior of others. Two forms of beliefs are introduced, one based on the state of the resource, the other on this state and on the others' strategy (their consumption). We focus on consistent equilibria, where beliefs must be consistent with observed past plays. Closed form expressions of the optimal policies are derived. Comparisons are made with the full information benchmark case. With strategy and state based beliefs, the agents may behave more (respectively, less) agressively than in the non-cooperative benchmark depending on the initial consumption level. When initial consumption is low, the optimal consumption path lies below that of the cooperative benchmark. We conclude the analysis by discussing the impact of public policies on the agents' choice of consumption patterns, and the robustness of the results for the case of renewable resources.
\end{abstract}

Keywords: Dynamic game; dynamic resource management; non probabilistic beliefs, conjectural variations.

\section{Introduction}

The interplay between external and strategic effects is a main feature of many economic issues. Non-cooperative behavior in the problem of resource management is shown to lead to overconsumption of the resource compared to a socially efficient (joint) management. Different studies have confirmed this issue in problems of resource management (Levhari and Mirman (1980), Fischer and Mirman (1992, 1996), or Houba et al (2000)). Several conclusions follow. Efficient consumption plans do exist, but this typically requires the use of discontinuous strategies (Dutta and Sundaram (1993)), or the threat 
of punishment (Benhabib and Radner (1992)). In this literature decision takers are assumed to be perfectly rational and to have perfect knowledge of the different characteristics of the setting. In other words, the information is assumed to be complete. In practice, agents might lack information regarding the other people's preferences, and their rationality might be bounded.

We revisit the problem of non-renewable resource management where information is incomplete, and consequently depart from the traditional complete information approach adopted in the above studies. In our procedure decision makers know the evolution rule of the resource but do not have information about their opponents' preferences and operate based on simple beliefs about their opponents' behaviour. An important feature is that players rely on nonprobabilistic beliefs, which we also refer to as conjectures (see for instance Figuières et al (2004)). Basically, players assume that a variation of their own consumption has a first order linear effect on the consumption of others.

There are different reasons for which we depart from the traditional Bayesian framework. The first one is that the Bayesian framework typically assumes the knowledge of the agents' utility functions and of the distribution of their potential types. In the present setting we want to analyse a situation where agents might not be perfectly rational. Moreover, they do not need knowledge of the utility function of others and of any distribution of types. The second reason is that empirical evidence is often inconsistent with the Bayesian framework. The notion of conjectures is used in the empirical literature as it seems to provide a general framework to model imperfect competition (see Slade (1995), Lopez de Haro et al. (2007) among other examples). As such it is often used to analyse oligopoly problems (Kalashnikov et al. (2009)) or environmental issues (problems of pollution control as in McKitrik (1999) for example). The theoretical literature on conjectural variation equilibrium has thus focused on several questions in order to provide theoretical foundations of this notion. A part of the literature tries to provide support for this notion in static games by introducing different forms of consistency. However, Lindh (1992) shows that endogeneizing conjectures by using notions of consistency does not rationalize conjectural equilibria in a static setting. The only way to rationalize this type of equilibrium is to develop a dynamic notion.

Another part of the literature focuses on the notion of conjectural variations in evolutionary or repeated settings. Some papers provide support for conjectural variations by considering evolutionary games with myopic agents and showing that consistent conjectures are evolutionary stable. This is shown by Muller and Normann (2005) in linear quadratic oligopoly models 
(and further generalized by Possajennikov (2009)). Dixon and Somma (2003) provide an evolutionary process converging to consistent conjectures in an oligopolistic model. Other papers consider a repeated game setting where dynamic notions of conjectural variations are introduced and analysed. Recent contributions are Friedman and Mezzetti (2002), and Jean-Marie and Tidball (2005), where the main focus is on repeated oligopoly games. Finally, learning procedures based on conjectures are developed in Jean-Marie and Tidball (2006) and Quérou and Tidball (2010). In these papers agents assume that a variation of their own strategy has a first order linear effect on the strategy of others, and beliefs are updated based on observations over time.

Still, it has been acknowledged that a fully consistent theory of conjectures should focus on models characterized by a dynamic evolution rule. The present paper focuses on such a situation and makes an interesting contribution to the literature by analysing a fully dynamic problem of natural resource management (fishery management is a good example). More specifically, both Friedman and Mezzetti (2002) and Jean-Marie and Tidball (2005) analyse purely repeated games. By contrast we analyze a discounted discretetime, infinite-horizon extraction game with conjectures, where the state of the resource is subject to a dynamic evolution rule and players use feedback strategies. Our main goal is to understand the influence of such simple beliefs on the agents' behavior. Specifically, we want to know if agents can learn to behave cooperatively in such a setting.

To our knowledge, this is the first contribution in this area that allows for a tractable analysis of the (optimal) consumption plans. Our contribution has two parts. First, we fix the issue of logical consistency by dealing with an explicitly dynamic notion of conjectures. Secondly, we show that agents can learn to behave cooperatively even though they rely on simple beliefs such as conjectures. Initial conditions (levels of consumption at the beginning of the process) are shown to play an important role in the agents' ability to adopt a cooperative behavior.

We consider two different types of beliefs, one where agents assume that the strategy of others is a function of the state of the resource at the previous period (state based beliefs), the other where the conjecture is a function of the state of the resource and of the other agents's strategy (state and strategy based beliefs). Beliefs are required to be consistent with observed 
policies (as in Jean-Marie and Tidball (2005)). ${ }^{1}$ Consistency requires that the paths corresponding to the players' beliefs must be consistent with observed past plays. Jean-Marie and Tidball (2005) show (in theorem 5.1) in a finite horizon, repeated game setting that, with state based beliefs ${ }^{2}$ the consistent equilibrium coincides with the feedback Nash equilibrium (under complete information). This result provides support (based on rational grounds) for this notion of consistency. We will prove (in proposition 4.1) that the same result is valid in the present setting (keeping in mind that we consider a fully dynamic game here). We will further develop the contribution by analysing the case of state and strategy based beliefs.

Let us describe the main parts of the analysis. The procedure and consistent solutions are defined. Convergence is studied, and the resulting policy is compared to the benchmark of complete information both from an economic and environmental point of view. It is proved that, depending on the agents' initial levels of consumption, there are situations where consistent solutions yield better outcomes in terms of environmental sustainability compared to the case of complete information Cournot Nash equilibrium. With strategy and state based beliefs, the agents behave more (respectively, less) agressively than in the non-cooperative benchmark when initial consumption is high (respectively, intermediate). When the initial consumption is low, the optimal consumption path lies below that of the cooperative benchmark. Finally, we discuss the impact that public policies (such as taxes) could have on the management of the resource in such a setting. Indeed such policies are usually present in problems of natural resource management. As such, they are to some extent part of the process, and it seems logical to discuss their impact on the analysis. We highlight that taxes could provide agents with appropriate incentives to decrease their initial level of consumption, so that the process generates a cooperative (or even more sustainable) management of the resource.

The above results show that agents relying on simple beliefs can learn to manage a natural resource cooperatively. However, this is shown to rely on the level of initial consumption that agents choose. This highlights how public policies could help decision making by ensuring that agents adopt the appropriate levels of initial consumption. These conclusions seem to us quite

\footnotetext{
${ }^{1}$ Other studies have defined notions of consistency in dynamic games, as Friedman (1977), Fershtman and Kamien (1985), or Laitner (1980). These notions raise issues regarding the computability of solutions. We may refer to Friedman and Mezzetti (2002) who propose another possible model too.

${ }^{2}$ The state refers to the usual definition when a repeated game is considered.
} 
consistent with the way people make decisions (history dependent policies, coexistence of different types of behavior). Firstly, path dependency has been highlighted by Bischi et al. (2005) in other situations as well (where agents are not perfecly informed about fish stocks). ${ }^{3}$ Secondly, the conclusions are interesting as well if one interprets the present process of forming conjectures as a potential heuristic that agents could decide to adopt to guide their decision making in such a setting. Indeed, the present process would enable them to avoid the burden of a more sophisticated analysis of strategic interactions.

The paper is organized as follows. The model and the main results of the benchmark case of full information are introduced in Sections 2 and 3 (respectively). The situations with state based and state and strategy based beliefs are analyzed in Section 4. In Section 5 we discuss the robustness of our results for renewable resources, the case of more than two agents, and the influence of initial consumption levels on the outcome of the process. Section 6 concludes. Technical details of one proof are provided in an appendix in Section 7 .

\section{The benchmark case of complete informa- tion}

The model of non-renewable resource extraction is briefly introduced in the first sub section. ${ }^{4}$ The players' utility functions and the specifics of the model are described and the notations explained.

\subsection{Dynamics}

We consider a two-agent problem of natural resource extraction, where the resource is subject to a dynamic evolution rule. The time horizon of the problem is infinite. Let $x_{t}$ be the stock of natural resource at time $t$. If $c_{i, t}$ denotes agent $i=1,2$ extraction at time $t$, then the evolution rule is given by

$$
x_{t+1}=x_{t}-c_{1, t}-c_{2, t}, \quad x(0)=x_{0} .
$$

\footnotetext{
${ }^{3}$ In practice, both the resource stock and the behaviour of others might be uncertain. Thus, both papers are useful complements since they tackle two complementary aspects of uncertainty.

${ }^{4}$ The problem has been analysed by Levhari and Mirman (1980) in the case of renewable resources. We will refer to this work in the present paper since this type of resource will be considered in Section 5 .
} 
We focus in Sections 3 and 4 on the case of a non renewable resource. This is made for analytical tractability (in order to derive closed form expressions of the optimal policies). In Section 5 we will discuss how the results extend to the case of renewable resources by relying on numerical works.

Let us discuss the assumptions of the present model. First, the biological rule and extraction technology are assumed to be linear. This is assumed in models of resource extraction to ensure that the situation remains analytically tractable. The two assumptions imply that a (unique) interior solution to the problem of optimal extraction will exist. ${ }^{5}$ In the present contribution this leads to the existence of an (unique) interior optimal consumption path. ${ }^{6}$ This enables us to make a precise comparison with the complete information model. ${ }^{7}$

Second, players share the same extraction technology. Again, this ensures that the optimal consumption policies can be characterized analytically. The present contribution constitutes the first application of the present notion of consistent beliefs to such dynamic models. Analytical results are required to highlight its main characteristics (link between initial conditions and optimal consumption policies, comparison with the benchmark case). Applying it to situations with heterogeneous agents is the next step of the project, but this will rely on a numerical approach.

In the next sub section the agents' utility functions are defined, and we elaborate on the implications resulting from this specification.

\subsection{The agents' payoff functions}

Each agent derives utility from present and future consumptions. A given agent's consumption policy influences obviously the consumption of the other by its influence on the evolution of the resource. As such we consider an infinite horizon dynamic game. As in the contribution of Levhari and Mirman (1980) we will assume that the relationship (for agent $i$ ) between consumption at period $t$ and the level of instantaneous utility derived is given by the following specification:

$$
u_{i}\left(c_{i, t}\right)=\log \left(c_{i, t}\right),
$$

\footnotetext{
${ }^{5}$ As said previously, we will discuss the case where the biological rule is non linear and concave in Section 5.

${ }^{6}$ When agents have perfect foresight, the existence of a Markov perfect equilibrium is ensured.

${ }^{7}$ Let us keep in mind that Levhari and Mirman (1980) do not analyse the case of a non renewable resource. We use the present specification as it is simple and will enable us to derive closed form expressions of the optimal policies.
} 
where $c_{i, t}$ denotes the consumption of agent $i$ at period $t$. We use such a specification for several reasons. Firstly, logarithmic utility functions are used frequently in problems of resource management (see, among other examples, a recent contribution by Koulovatianos et al. (2009) on optimal growth and learning). Secondly, this specification will enable us to derive closed-form expressions of the optimal consumption plans and of the state dynamics in the present setting. This is consistent with the main goal of the present paper, which is to highlight the main characteristics of the process introduced in Section 4 (in terms of the economic efficiency of the consumption plan and of the sustainability of the management of the resource) by comparing our findings with those of the benchmark case in terms of the long run management strategies that result from each contribution.

Agents are assumed to choose their optimal consumption policies in order to maximize the discounted sum of their instantaneous utility derived from consumption, that is, to maximize

$$
\sum_{t=0}^{\infty} \beta^{t} u_{i}\left(c_{i, t}\right),
$$

taking into account the evolution of the resource (and the influence of the other agents' consumption strategy on this evolution as well). In the above specification of payoffs we assume that $0<\beta<1$ denotes the measure of the agents' time preferences. In the case of Levhari and Mirman (1980) the information is assumed to be complete, while, in the present contribution, agents do not know their opponent's utility function and form beliefs about their behavior. We will later assume two simple forms of such beliefs and we will solve for the above specification of the dynamic game.

In the next sections we will characterize the optimal policies corresponding to the complete information benchmark case, ${ }^{8}$ then we will define the notions of state based and strategy and state based beliefs (as introduced in Jean-Marie and Tidball (2005)) and we will apply these notions to the present model. This will enable us to derive comparisons between our findings and the benchmark case.

\section{The full information benchmark case}

We first consider the complete information benchmark case. We characterize the optimal consumption policies (and related state dynamics) under joint

\footnotetext{
${ }^{8}$ Because this characterization is straightforward, we will omit the full proofs.
} 
and non-cooperative managements (in sections 3.1 and 3.2 respectively).

\subsection{The cooperative case}

In this section the information is assumed to be complete. A given agent's problem is:

$$
\max _{\left\{c_{1, t} ; c_{2, t}\right\}} \sum_{t=0}^{\infty} \beta^{t}\left[\log \left(c_{1, t}\right)+\log \left(c_{2, t}\right)\right]
$$

subject to

$$
x_{t+1}=x_{t}-c_{1, t}-c_{2, t}, \quad x_{0} \text { given. }
$$

We restrict to feedback strategies (we assume that $c_{i, t}=a_{i} x_{t}$ ) for any player $i=1,2$. Plugging this expression into the above equality, we obtain

$$
x_{t+1}=\left(1-a_{1}-a_{2}\right) x_{t}, \quad x_{t}=\left(1-a_{1}-a_{2}\right)^{t} x_{0} .
$$

Solving the problem for $a_{1}$ and $a_{2}$, the optimal consumption policies are:

$$
a_{1}=a_{2}=(1-\beta) / 2, \quad x_{t}^{c}=\beta^{t} x_{0}, \quad c_{i, t}^{c}=\frac{1-\beta}{2} \beta^{t} x_{0},
$$

where $c_{i, t}^{c}$ denotes agent $i$ 's optimal consumption policy (at period $t$ ) under joint management.

\subsection{The non-cooperative case: Nash equilibria}

For players $i=1,2$, the problem is specified as follows:

$$
\max _{\left\{c_{i, t}\right\}} \sum_{t=0}^{\infty} \beta^{t} \log \left(c_{i, t}\right)
$$

subject to

$$
x_{t+1}=x_{t}-c_{1, t}-c_{2, t}, \quad x_{0} \text { given. }
$$

We consider feedback strategies again. The problem becomes:

$$
\max _{\left\{a_{i}\right\}} \sum_{t=0}^{\infty} \beta^{t}\left[\log \left(a_{i}\right)+t \log \left(1-a_{1}-a_{2}\right) .\right]
$$

Solving for $a_{1}$ and $a_{2}$, we obtain the following non-cooperative consumption policies:

$$
a_{1}=a_{2}=\frac{1-\beta}{2-\beta}, \quad x_{t}^{N}=\left(\frac{\beta}{2-\beta}\right)^{t} x_{0}, \quad c_{i, t}^{N}=\frac{1-\beta}{2-\beta}\left(\frac{\beta}{2-\beta}\right)^{t} x_{0},
$$


where $c_{i, t}^{N}$ denotes the optimal consumption policy of agent $i$ (at period $t$ ) under non-cooperative management of the resource. Notice that

$$
x_{t}^{c}=\beta^{t} x_{0}>\left(\frac{\beta}{2-\beta}\right)^{t} x_{0}=x_{t}^{N}, \quad \forall t .
$$

Strategic considerations lead to a more intensive extraction of the resource than under joint management. This is the main conclusion provided by Levhari and Mirman (1980) in the case of a renewable resource.

We would like to stress the following point. We could have used the Bellman equation associated to the above non-cooperative problem in order to solve for the optimal consumption policy at each period of the game. Instead we use the linear structure of the game. The same method will be used in Section 4.1 to derive the consistent solution with state based beliefs when information is incomplete.

\section{The situation of incomplete information with conjectures}

In this section we consider that agents know the evolution rule of the resource but have limited information on the other agent's preferences. They form conjectures on their opponent's behavior. If one interprets an agent's conjecture as his type, then the type describes this agent's belief about the nature of the strategic interaction with his opponent. By contrast with the Bayesian framework, no knowledge about the other agent's form of utility function or about the distribution of types is needed.

We first analyse the case where players use state-based beliefs, that is, where beliefs are a function of the remaining stock of the resource at any given period (in section 4.1). We confirm the result of Jean-Marie and Tidball (2005, theorem 5.1) who show (in a repeated game setting) that the consistent equilibrium with state based conjectures coincides with the complete information feedback Nash equilibrium. Then we analyse the case of strategy and state based beliefs (in section 4.2) and we compare the optimal consumption policies (corresponding to the consistent solution) with those of the full information benchmark case. 


\subsection{The case of state based beliefs}

Let us consider in a first step the situation where any player $i$ conjectures that player $j$ 's consumption policy at period $t$ is a function of $x_{t}$ only. Specifically, we consider that agent 1's beliefs regarding the behavior of agent 2 have the following form:

$$
c_{2, t}^{1}=a_{2} x_{t} .
$$

In other words, agent 1 conjectures that the optimal consumption of agent 2 at any given period $t$ is a function of the stock of the resource at the beginning of the same period. It is implicitly assumed that each agent can observe the stock of the resource at the end of each period.

Now we look for consistent solutions, that is, solutions such that the conjectured policy corresponds to the actual optimal policy. This means that conjecture $a_{2}$ must be such that the conjectured value of consumption of agent 2 (as implied by the above expression) corresponds to the solution to the optimal problem of agent 2 (for any given period).

It can be checked that a straightforward application of this definition of consistency leads to the following conclusion. ${ }^{9}$

Proposition 4.1. Let us denote by $c_{i, t}^{f c}$ the (feedback) consistent optimal consumption policy of agent $i(i=1,2)$ at period $t$. When agents use state based beliefs, the optimal consumption policy is characterized, for any period $t$ and any agent $i$, by:

$$
c_{i, t}^{f c}=c_{i, t}^{N},
$$

where $c_{i, t}^{N}$ denotes the non-cooperative optimal consumption policy in the benchmark (full information) case.

The proof is omitted as it is immediately checked that one has to solve the same system of first order conditions in both situations. Because of the assumptions there is a unique optimal policy that satisfies these conditions, which yields the conclusion.

The implication of this result is that the non-cooperative management of the resource is equivalent under both complete and incomplete information. Even though this conclusion might be perceived as disappointing at first sight, there is a notable difference between the two cases. Indeed, requiring consistency enables to get rid of the assumption of perfect knowledge about

\footnotetext{
${ }^{9}$ We use the specific structure of the game as it enables us to simply identify the optimal coefficients characterizing the solution.
} 
the agents' utility functions. Thus, the case of state based beliefs provides some support for the notion of consistent conjectures. In this simple case conjectures are rationalized in that the notion of consistency leads to the well know concept of feedback Nash equilibrium.

We will now introduce a second type of beliefs, which are defined as a function of the state of the resource and of the agents' consumption strategy. We will then compare the optimal consumption policies with those of the benchmark case.

\subsection{The case of state and strategy based beliefs}

We first consider a type of beliefs where a conjecture is a function of both the state of the resource and of the consumption pattern. We will see that this definition allows for a rich pattern of potential behaviors. Moreover it will be stressed that the optimal consumption policy (and thus the management of the resource along the equilibrium path) depends on the initial consumption levels. This influence will be characterised as well.

We now proceed with the definition of strategy and state based beliefs. Let us consider the case of player 1 . This player conjectures that player 2's consumption decision at period $t$ is given by:

$$
c_{2, t}^{1}=a_{2} x_{t}+b_{2} c_{1, t-1},
$$

where $a_{2}$ and $b_{2}$ model the player's beliefs. In other words, agent 1 assumes that the consumption policy of agent 2 at period $t$ is a function of the state of the resource at period $t$ and of agent 1's consumption strategy at period $t-1 .^{10}$

This form deserves further comment. Firstly, it is implicitly assumed that agents observe the level of the resource and the consumption of others at every period. Secondly, it is worth explaining why we choose this form. This is the simplest form of beliefs that can be thought of when beliefs are defined as a function of the characteristics of the situation at each period, namely, the state of the resource and the strategy of the other agents. Since agents are assumed to learn the behavior of others, it seems natural to assume that they define their beliefs as a function of the only characteristic of the other

\footnotetext{
${ }^{10}$ One should keep in mind that $x_{t}$ is the state of the resource at the beginning of period $t$. As such, it is observable by the agent before he chooses his optimal consumption policy at this period, together with consumptions at period $t-1$. Thus, his belief at period $t$ is based on the data that are observable at the beginning of this period.
} 
agents that they can observe. We consider the simplest form of relationship to define conjectures by assuming that the consumption of one agent has a first order linear effect on the behavior of the others. A natural implication is that agents are (to some extent) boundedly rational. The other way to think about the definition of the conjectures is to consider the process of forming conjectures as a potential heuristic that agents could decide to adopt to guide individual decision making. With this perspective in mind, the process could be useful if it proves to be reasonably efficient. We will come back to this question during the analysis of the process. ${ }^{11}$

Let us come to the notion of feedback consistency. If there is a persisting difference between the consumption of player 2 as conjectured by player 1 and the actual optimal policy of player 2 , then player 1 would have incentives to stop relying on this scheme. This is why we require that solutions be consistent. Consistent solutions are such that the conjectured policy corresponds to the actual optimal policy. This notion of consistency enables us to introduce an equivalent formulation of the problem. ${ }^{12}$

Specifically, from Jean-Marie and Tidball (2005) it is known that finding the feedback consistent solution to the present problem is equivalent to finding the solution to:

$$
\max _{\left\{c_{t}^{i}\right\}} \sum_{t=0}^{\infty} \beta^{t} \log c_{i, t}
$$

subject to the following constraints:

$$
x_{t+1}=x_{t}-a_{j} x_{t}-b_{j} y_{t}-c_{i, t}
$$

and

$$
y_{t+1}=c_{i, t}
$$

where initial stock $x_{0}$ and catch $c_{i,-1}$ are common knowledge at the beginning of the problem. ${ }^{13}$ Variable $y_{t}$ is introduced as a second state variable, since this new formulation will be useful to solve the problem. As an example, for $i=1$, the dynamics of the equivalent problem can be rewritten as follows:

$$
x_{t+1}-x_{t}=-a_{2} x_{t}-b_{2} y_{t}-c_{1, t}
$$

\footnotetext{
${ }^{11}$ Let us notice that the notion of strategy and state based beliefs shares a feature in common with the notion introduced by Friedman and Mezzetti (2002). Specifically, they use the other agents' strategies at period $t-1$ in order to define the agents' conjectures at period $t$. We will see that the present paper is consistent with their analysis, as we will check that there is a link between the initial conditions (the consumption levels) and the optimal consumption path.

${ }^{12}$ We elaborate on the characterization of consistent solutions by using the equivalent formulation of the problem.

${ }^{13}$ Knowledge of $c_{i,-1}$ is equivalent to knowing $c_{i, 0}$ in the present setting because of the consistency requirement.
} 
and

$$
y_{t+1}-y_{t}=c_{1, t}-y_{t} .
$$

In the present case the state vector of the dynamics is defined by equations (3) and (4). Specifically, at any period $t$ the state of the process is given by $\left(x_{t}, c_{1, t-1}, c_{2, t-1}\right)$. Requiring feedback consistency amounts to requiring that the functional relationship that links $\left(x_{t}, c_{1, t-1}, c_{2, t-1}\right)$ to the belief of each agent regarding the opponent's optimal catch at period $t$ corresponds to the actual optimal policy of this country.

From this definition of consistency the remaining steps in calculations are straightforward. When we derive the optimality conditions associated to the above problem, we will obtain a set of parameters $\left(a_{2}, b_{2}\right)$ (respectively, a set of pairs $\left(a_{1}, b_{1}\right)$ for agent 2$)$ that are potential solutions. Then we will look for the specific values that correspond to the feedback consistent solutions. The above specification of the problem will enable us to derive the closed form expression of the optimal consumption path. Then we will compare the optimal policies with those of the benchmark case provided in Section 3. Specifically, we will prove that the symmetric consistent optimal consumption policy (and resulting stock level) is characterized by

$$
c_{t}^{f c}=[\beta(1-a)]^{t} c_{0}, \quad x_{t}^{f c}=\frac{c_{t}^{f c}-b c_{t-1}^{f c}}{a},
$$

for any period $t$ ( $c_{t}^{f c}$ denotes the symmetric (feedback) consistent optimal consumption policy at period $t$ with strategy and state based beliefs).

We can now state the main result of this section. We provide an explicit link between the level of initial consumption and the closed form expressions of optimal consumption policies and corresponding state dynamics. This enables us to compare the different policies to the benchmark cases of sections 3.1 and 3.2. ${ }^{14}$ The following remark will prove to be useful in this analysis.

Remark 4.1. First notice that we deal with values of $c_{0}$ such that $x_{0}>$ $2 c_{0}$, otherwise the stock will be exhausted before the beginning of the process. Second, one could notice that, since the discount factor $\beta$ lies between 0 and 1, we have the following inequalities:

$$
\frac{1-\beta}{2} x_{0}<\frac{1-\beta}{2-\beta} x_{0}<\frac{1}{2} x_{0} .
$$

These inequalities will be useful in the next result.

\footnotetext{
${ }^{14} \mathrm{~A}$ general conclusion will be that the resource will be exhausted eventually (since optimal consumption is positive and the resource is non renewable). The important point is to compare the findings under incomplete and full information.
} 
The main conclusions are described as follows.

Proposition 4.2. The following results hold:

- If $c_{0}=\frac{1-\beta}{2} x_{0}$, then $a=0$ and $b=\beta$. The feedback consistent solution is given by:

$$
c_{t}^{f c}=\beta c_{t-1}^{f c}, \quad x_{t}^{f c}=\beta^{t} x_{0} .
$$

In this case $x_{t}^{f c}=x_{t}^{c}$. In others words, if the level of initial consumption corresponds to that of the full information cooperative case, then the feedback consistent solution coincides with the benchmark solution under joint management.

- If $c_{0} \neq \frac{1-\beta}{2} x_{0}$ then :

$$
c_{t}=\left[1-2 \frac{c_{0}}{x_{0}}\right]^{t} c_{0}, \quad x_{t}=\left[1-2 \frac{c_{0}}{x_{0}}\right]^{t} x_{0} .
$$

Moreover, we can state the following comparisons:

- if $c_{0}<\frac{1-\beta}{2} x_{0}$ then $a<0$ and $b>0$ and $x_{t}^{f c}>x_{t}^{c}>x_{t}^{N}$;

- if $\frac{1-\beta}{2} x_{0}<c_{0}<\frac{1-\beta}{2-\beta} x_{0}$ then $a>0, b>0$ and $x_{t}^{N}<x_{t}^{f c}<x_{t}^{c}$;

- if $\frac{1-\beta}{2-\beta} x_{0}<c_{0}<\frac{1}{2} x_{0}$ then $a>0$ and $b<0$ and $x_{t}^{f c}<x_{t}^{N}<x_{t}^{c}$.

Proof. See Appendix.

Let us briefly elaborate on the above result. The optimal consumption policies (and thus, the stock of the resource along the equilibrium path) depend on the initial consumption level. This is an important feature of the present model. The observation of this initial condition can help one to anticipate which of the patterns of consumption will prevail. From another perspective, this implies that one might influence the consumption path that will be chosen by focusing on the initial level of consumption.

Remark 4.2. There is an interesting interpretation of Proposition 4.2. ${ }^{15}$ The form of the beliefs $c_{j, t}^{i}=a_{j} x_{t}+b_{j} c_{i, t-1}$ can be thought about as follows. As player $i$ consumes more today, he believes that the other agent will react and increase his own consumption tomorrow. Thus, agent $i$ will be punished next period by the other player's faster extraction. This creates a situation close to that of tit-for-tat strategies (even though information is incomplete in the present setting), which might enable the players to sustain the full information cooperative path or more environmentally sustainable paths (depending on the initial consumption levels).

\footnotetext{
${ }^{15}$ We are grateful to a referee for providing this interpretation.
} 


\subsection{Discussion}

Proposition 4.2 leads to several predictions. The first part of the result states that the feedback consistent solution coincides with the cooperative solution under complete information provided that the initial level of consumption is the same in both cases. This is intuitively appealing. If agents cooperate initially (even though they might not know it due to incomplete information) then asking for consistency ensures that cooperation will be sustained in the long run.

If the initial consumption is too high, then the present procedure leads to a more aggressive pattern than even in the non-cooperative case under full information. In such a case the effects of strategic behaviors are reinforced by incomplete information. However, provided that agents' consumption level lies initially below a threshold value the previous conclusion is reversed. Specifically, for moderate values, the stock of the resource lies in between non-cooperative and joint management patterns under full information.

When initial consumption is sufficiently low, we obtain the surprising conclusion that the present procedure leads to an under-exploitation of the resource compared to the full information cooperative benchmark (if one focuses on economic efficiency only, that is, on getting the most utility out of consumption). The intuition is as follows. Unlike the situation of complete information, the choice of the initial consumption level influences the shape of the optimal consumption path. The consistency requirement enables one to select a pattern of individual behavior that comes close to pure cooperation. Then, the choice of a sufficiently low initial consumption level enables the agents to select a path that is parallel to the full information cooperative benchmark, but that lies below it (due to the choice of a lower initial consumption level). This result has an interesting implication. There are situations where the management of the resource could be subject to other criteria than economic efficiency. For instance, it could be the case that the focus be on sustainable management. In such a case, the process of forming conjectures would help agents to learn to coordinate on a management path which would be even more appropriate (with regard to sustainability) than the cooperative benchmark. This suggests that in the third case incomplete information makes the emergence of alternative (and more cautious) patterns of behavior plausible, and our procedure takes advantage of this possibility.

There is a final insight to be noticed. In many problems of resource management public policies (taxes, quotas) are either implemented or potentially 
available. Thus, these policies can be considered as part of the setting. Parts of Proposition 4.2 have implications for the use of such instruments. Indeed, it is shown that a more efficient management of the resource (compared to the non cooperative benchmark) is feasible provided that initial consumption is kept below the level that corresponds to a non cooperative management under complete information. The sustainability of the resource can be further improved if initial consumption is lower than in the cooperative benchmark. Such low levels of initial consumption can be difficult to implement, and it could be the case that some (public) intervention be required to achieve this task. It is easy to understand that an appropriate extraction tax would then contribute to a better management outcome, as it would lead to a decrease in the level of initial consumption, which would then ensure that the agents would learn to adopt a more cooperative pattern of behavior.

\section{Extensions}

In the analysis we focus on a quite simple case in order to derive closed form expressions of the optimal consumption policies and of the resulting evolution of the stock of the resource. Specifically, we consider a non-renewable resource, and we study the case where there are only two agents. In this section, we first discuss these assumptions. Secondly, we analyse how agents may possibly choose their initial consumption levels.

\subsection{The case of renewable resources}

In Proposition 4.2 we characterize the optimal consumption path under consistent conjectures. In order to obtain this characterization, we assume that the resource is non renewable. It might be interesting to discuss whether one might expect (from a qualitative point of view) a similar type of result in the case of renewable resources. Thus, we consider the following biological rule:

$$
x_{t+1}=\left[x_{t}-c_{1, t}-c_{2, t}\right]^{\alpha}, x(0)=x_{0},
$$

where $\alpha \in(0,1)$ denotes the rate of regeneration of the resource. All other specifications and notations remain the same. We focus here on one of the most interesting cases described in Proposition 4.2. Specifically, provided that agents choose an initial consumption level corresponding to cooperative management, the process results in the cooperative consumption path. As a reminder (Levhari and Mirman (1980)), the full information cooperative solution is characterised (for any period $t$ ) by:

$$
c_{t}^{c}=\frac{1}{2}(1-\alpha \beta) x_{t}^{c}, x_{t+1}^{c}=\left(\alpha \beta x_{t}^{c}\right)^{\alpha} .
$$


Now, regarding our process, the expressions of the state variables and of the Hamiltonian function associated to agent $i$ 's problem becomes:

$$
\begin{gathered}
x_{t+1}-x_{t}=\left[\left(1-a_{j}\right) x_{t}-c_{i, t}-b_{j} y_{t}^{i}\right]^{\alpha}-x_{t}, \\
y_{t+1}^{i}-y_{t}^{i}=c_{i, t}-y_{t}^{i}
\end{gathered}
$$

and

$$
H=\beta^{t} \log c_{i, t}+\Pi_{t}^{i}\left[x_{t+1}-x_{t}\right]+\lambda_{t}^{i}\left[y_{t+1}^{i}-y_{t}^{i}\right]
$$

where $\Pi_{t}^{i}$ and $\lambda_{t}^{i}$ are the associated adjoint variables. This is a well-defined problem. Optimal consumption policies exist and satisfy, for any period $t$ :

$$
\begin{aligned}
\frac{\partial H}{\partial c_{i, t}}=0 & =\frac{\beta^{t}}{c_{i, t}}-\Pi_{t}^{i} \alpha\left[\left(1-a_{j}\right) x_{t}-c_{i, t}-b_{j} y_{t}^{i}\right]^{\alpha-1}+\lambda_{t}^{i}, \\
\Pi_{t-1}^{i} & =\prod_{t}^{i} \alpha\left[\left(1-a_{j}\right) x_{t}-c_{i, t}-b_{j} y_{t}^{i}\right]^{\alpha-1}\left(1-a_{j}\right),
\end{aligned}
$$

and

$$
\lambda_{t}^{i}=-\Pi_{t}^{i} b_{j} \alpha\left[\left(1-a_{j}\right) x_{t}-c_{i, t}-b_{j} y_{t}^{i}\right]^{\alpha-1} .
$$

The second condition can be rewritten as:

$$
\Pi_{t-1}^{i}=-\lambda_{t-1}^{i} \frac{\left(1-a_{j}\right)}{b_{j}} .
$$

The non-linear evolution of the resource does not enable us to provide a closed form expression of the optimal consumption policies. Nonetheless, we know that these policies are characterized by the above conditions and the consistency requirement (for $i, j=1,2$ and any period $t$ ):

$$
c_{j, t}=a_{j} x_{t}+b_{j} c_{i, t-1} .
$$

As a result, we adopt the following steps. First, we ask whether, for any rate of renewal $0<\alpha<1$ and an initial consumption (corresponding to the cooperative solution), the consistent process generates a consumption path that approximates the full information cooperative solution. In order to answer this question, we rely on numerical simulations. Since we cannot provide closed form expressions, we generate numerically a trajectory that will approximate the consistent optimal policies (with respect to an approximation parameter $\varepsilon$ chosen sufficiently small). Then, we check if the generated policy constitutes a good approximation of the cooperative solution.

Let us focus on approximating the consistent optimal policies. The numerically generated policy relies on a certain number of parameters, namely $a$, 
$b$ and $\Pi_{0}$. The first thing to notice is that if the policy is a good approximation, then there will be consistency with respect to the steady state of the resource. This implies that $a$ and $b$ satisfies a first condition so that consistency holds. Now, in order to run the simulations, we have to initialize the process. Anticipating our claim of cooperation and hopping that it will be a good approximation of consistency, we consider the following way to do so. We require that the initial consumption level coincides with the initial full information cooperative level. We use $\alpha=0.5, \beta=0.8$ and $x_{0}=0.5$ (the initial cooperative consumption is $c_{0}^{c}=0.15$ ). Figure 1 highlights that a good approximation to the consistent trajectory is obtained. Indeed, the variation $c_{t}-\left(a x_{t}+b c_{t-1}\right)$ becomes small after ten time periods.

\section{Figure 1 around here.}

We are now in a position to assess whether our generated $\varepsilon$-consistent trajectory constitutes a good approximation to the full information cooperative path. Figure 2 (below) illustrate that the first part of our results are robust to the case of renewable resources. Specifically, when initial consumption corresponds to cooperative management under complete information, the consistent process constitutes a good approximation of the entire cooperative path.

\section{Figure 2 around here.}

In a second step, we ask whether the second part of our results in Proposition 4.2 are also robust. In order to illustrate this feature, we generate policies where the initial consumption is allowed to vary. The last graph highlights the robustness of the properties obtained in Proposition 4.2. Specifically, when the initial consumption level is higher (respectively, lower) than at the cooperative solution, agents consume more (respectively, less) than on the cooperative path.

\section{Figure 3 around here.}

In the above graph we set $c_{0}=0.18>c_{0}^{c}=0.15$, and we obtain that the stock of the resource resulting from the process remains lower than that of the full information cooperative path. Indeed, we notice that the corresponding line lies below that of the cooperative solution.

To summarize, simulations seem to suggest that the results obtained in the present paper exhibit some robustness when non-linearity (of the evolution of the resource) is taken into account. 


\subsection{The case of more than two agents}

The main results (namely Propositions 4.1 and 4.2) have been proved in the case of two agents. We now discuss whether these results are robust when one considers multilateral interactions.

In order to highlight the robustness, we define the form of the conjectures that will be used when there are more than two agents. We will focus on the case of three players so that the exposition is kept as simple as possible.

In the case of state-based beliefs, the form of the conjectures can account for more than one opponent as long as one forms conjectures on the other agents as an aggregate. Let us define agents' conjectures as follows:

- Agent 1 conjectures that

$$
c_{2, t}+c_{3, t}=c_{-1, t}^{1}=a_{-1} x_{t}
$$

- Agent 2's conjecture is

$$
c_{1, t}+c_{3, t}=c_{-2, t}^{2}=a_{-2} x_{t}
$$

- The third agent conjectures that

$$
c_{1, t}+c_{2, t}=c_{-3, t}^{3}=a_{-3} x_{t} .
$$

With this definition, it is straightforward to check that, focusing on the corresponding first order conditions, the consistency requirement will result in the following equalities:

$$
\frac{1}{c_{1, t}^{f c}}=\frac{1}{c_{2, t}^{f} c}=\frac{1}{c_{3, t}^{f c}} .
$$

The first order conditions will then be identical to that of non-cooperative management, resulting in the following consumption policy for any agent $j$ :

$$
c_{j, t}=\frac{1-\delta}{3-2 \delta} x_{t} .
$$

This trajectory coincides with the non-cooperative consumption path. This is the same result as in Proposition 4.1.

Regarding the case of state and consumption-based beliefs, the form of conjectures remains unchanged, the only amendment being that agents form 
conjectures on the extraction of the other two agents considered as an aggregate. In this case, focusing on the case of agent 1 (as an example), feedback consistency would require that we obtain at every period $t$ :

$$
a_{-1} x_{t}+b_{-1} c_{1, t-1}=c_{2, t}+c_{3, t} .
$$

In the symmetric case where $c_{1,0}=c_{2,0}=c_{3,0}=c_{0}$ and focusing on the symmetric solution, the above condition would be rewritten as:

$$
a x_{t}+b c_{t}=c_{2, t}+c_{3, t}=2 c_{t} .
$$

With this amendment in mind, the proof of Proposition 4.2 remains entirely valid. One derives the following characterisation of parameters $a$ and $b$ in the case of three agents:

$$
a=\frac{x_{0} \beta-x_{0}+3 c_{0}}{x_{0} \beta}, b=\frac{\left(x_{0}-3 c_{0}\right)\left[2 c_{0} \beta-3 c_{0}-x_{0} \beta+x_{0}\right]}{\beta c_{0} x_{0}},
$$

and Proposition 4.2 generalises to the present setting. For instance, in the second case, the stock dynamics is characterised as $x_{t}=\left(1-3 \frac{c_{0}}{x_{0}}\right)^{t} x_{0}$, and the comparison with respect to non cooperative management and the cooperative solution remains the same.

\subsection{On the influence of initial consumption}

Proposition 4.2 highlights what we consider as an interesting feature of the process: the optimal consumption path depends on the initial condition. To us, this is not a weakness of the process as there are many real world economic instances that exhibit path dependency. Moreover, it highlights a potential role for public intervention, which is to influence initial consumption so that the process could then lead the agents to the desired optimal path.

It might still be interesting to study if one can provide support to the choice of certain initial consumption levels. First, using the results obtained in Section 4, it is easily checked that the agents' payoffs can be expressed as functions of the initial consumption levels. Specifically, let us denote $c_{i, 0}$ agent $i$ 's initial consumption level. The agents' consumption policies can then be derived as:

$$
c_{i, t}=\beta^{t}\left(\frac{x_{0}-c_{1,0}-c_{2,0}}{\beta x_{0}}\right)^{t} c_{i, 0} .
$$

Plugging this expression into the agents' payoffs, we obtain:

$$
f_{i}\left(x_{0}, c_{1,0}, c_{2,0}\right)=\sum_{t=0}^{\infty} \beta^{t} \ln \left(\beta^{t}\left(\frac{x_{0}-c_{1,0}-c_{2,0}}{\beta x_{0}}\right)^{t} c_{i, 0}\right) .
$$


Rewriting, we get:

$$
f_{i}\left(x_{0}, c_{1,0}, c_{2,0}\right)=\frac{\ln \left(c_{i, 0}\right)}{1-\beta}+\left[\ln \beta+\ln \left(\frac{x_{0}-c_{1,0}-c_{2,0}}{\beta x_{0}}\right)\right] \frac{\beta}{(1-\beta)^{2}} .
$$

Thus, anticipating the use of the process, we can rewrite the agents' payoffs as an instantaneous function of the initial consumption levels. How can one provide support to specific levels of initial consumption? One might be tempted to consider a one-shot game (before the agents play according to this process) where agents would play either a la Nash or cooperatively, and then solve the corresponding problem. It is easily checked that initial consumption would correspond either to the Nash equilibrium of the game, or to the initial full information cooperative consumption level. However, one should be cautious when providing support to initial consumption levels.

Indeed, agents are assumed to form conjectures in the present paper because they do not know their opponent's utility function. As such, one has to consider that they form conjectures on the other agent's choice of initial consumption too. Moreover, since the problem is now related to instantaneous payoff functions, one has to rely on a different form of conjectures than that used in the present paper. We are going to use a process of forming conjectures introduced in Jean-Marie and Tidball (2006) that is suited to the new specification of the payoff functions.

We refer to Jean-Marie and Tidball (2006, Section 2) for a complete and formal definition of the process that we use. Agents now define their conjecture regarding the opponent's choice of initial consumption with respect to an exogenously given pair of reference consumption levels $\left(\overline{c_{1,0}}, \overline{c_{2,0}}\right)$. This reference point might, for instance, correspond to a status quo ${ }^{16}$ The form of the first agent's conjecture is thus:

$$
c_{2,0}^{1}=\overline{c_{1,0}}+r_{2}\left(c_{1,0}-\overline{c_{1,0}}\right),
$$

where $r_{2}$ denotes the agent's conjecture. Agent 2 adopts a similar form of conjecture regarding the other agent's behavior. It amounts to assuming that an agent conjectures that his initial consumption choice has a first order effect on the initial consumption of his opponent. Plugging this expression into the agents' payoffs, we obtain:

$f_{i}\left(x_{0}, c_{i, 0}, c_{j, 0}^{i}\right):=f_{i}\left(c_{i, 0}, c_{j, 0}^{i}\right)=\frac{\ln \left(c_{i, 0}\right)}{1-\beta}+\left[\ln (\beta)+\ln \left(\frac{x_{0}-c_{i, 0}-\overline{c_{i, 0}}-r_{j}\left(c_{i, 0}-\overline{c_{i, 0}}\right)}{\beta x_{0}}\right)\right] \frac{\beta}{(1-\beta)^{2}}$.

\footnotetext{
${ }^{16}$ We refer to Jean-Marie and Tidball (2006) for a discussion of this assumption.
} 
The process developed in Jean-Marie and Tidball (2006) is based on learning by trial and error. The agents' conjectures $r_{1}$ ad $r_{2}$ are updated until they become stationary as each agent $i$ tries to make the best choice regarding $c_{i, 0}$ (anticipating that it will be used as the initial consumption level when agents will play according to the process defined in this paper). We propose to check if one can provide support to the choice of certain values of initial consumption levels as the stationary outcomes of the learning process developed in Jean-Marie and Tidball (2006).

In order to obtain the stationary outcomes of this process, one has to derive the first order conditions (for given conjectures $r_{1}$ and $r_{2}$ ) related to the utility functions $f_{i}\left(c_{i, 0}^{\prime} c_{j, 0}^{i}\right)$, which enables one to express each agent's optimal choice as a function of the agents' conjectures. Then each agent updates his own conjecture by trial and error until it converges eventually. The stationary values of the conjectures are characterised as fixed points of the updating process. Using the result provided in Jean-Marie and Tidball (2006, Section 3) there are two pairs of stationary values of the agents' conjectures in the present problem:

1. The first pair satisfies $r_{1}=r_{2}=1$, and this pair corresponds to the following choices:

$$
c_{1,0}=c_{2,0}=\frac{1}{2}(1-\beta) x_{0} .
$$

Thus, the initial consumption level of the full information cooperative solution path is supported as a stationary outcome of the process.

2. The expression of the second solution depends in a complex way on the parameters $\overline{c_{i, 0}}$. If one looks for the symmetric solution where $\overline{c_{1,0}}=\overline{c_{2,0}}=\overline{c_{0}}$, it follows that it is characterised as, for any agent $i$ :

$$
c_{i, 0}=\overline{c_{0}},
$$

that is, the consumption levels chosen correspond to those provided by the reference point.

Now, if one looks for predictions that are robust to the specification of the process of forming initial conjectures (to any specification of the reference point $\left(\overline{c_{1,0}}, \overline{c_{2,0}}\right)$ ), then the first pair of initial consumption levels is the only feasible solution. Thus, using the process developed in Jean-Marie and Tidball (2006) as a way to assess which one of the possible outcomes in Proposition 4.2 is a preferred equilibrium, one can conclude that the full information initial consumption level is supported as a preferred choice. 


\section{Concluding remarks}

We study a problem of resource management under incomplete information when decision takers interact strategically and where each agent's consumption decision has an influence on the evolution of the size of the resource. A learning procedure is developed where agents form conjectures which are updated according to available observations. We consider two types of conjecture, one based only on the state of the resource, the other based on the state and the consumption strategy of the other agents. The solution studied is such that beliefs must be consistent with observed behaviors.

Closed form expressions of the optimal policies are obtained and compared to the benchmark case (as provided in Section 3). It is proved that the optimal policies correspond to the full information, non-cooperative solution when conjectures are based on the state of the resource only. In the second case (with state and strategy based beliefs), the consistent solution is shown to yield better outcomes regarding the resource management in the long run compared to joint management if initial consumption is sufficiently low, or to lead to a more aggressive pattern than the non-cooperative benchmark if initial consumption is too high. For intermediate values of initial consumption, the optimal path lies in between the non-cooperative and cooperative benchmark cases. Finally, several extensions (more than two agents, renewable resources, selection of specific initial consumption levels) are discussed.

From a practical point of view, the present procedure provides an explicit link between the initial conditions and the resulting long run policies. In other words, the dependence of convergence on the initial conditions explains which of the equilibria will prevail given the initial conditions. As incomplete information is a usual characteristic of resource extraction, the knowledge of an explicit link between initial consumption and resulting long run dynamics is a potentially useful property. If one thinks about this process of forming conjectures as a potential heuristic that agents could decide to adopt to guide individual decision making, then this link is useful as it provides a way to induce agents to adopt different patterns of management of the resource.

Even though the present contribution highlights some interesting properties of the learning procedure, we abstract from several issues. A first extension would be to analyse cases where agents could be heterogeneous. For instance, they could use different extraction technologies. The investigation of other forms of conjectures might be interesting in the case of renewable resources. These points are left for future research. 


\section{Appendix}

In this appendix we now provide the proof of proposition 4.2. We proceed in three steps. First, we derive the optimality conditions associated to the problem. This enables us to characterize the optimal consumption path. Secondly, we characterize the solutions that satisfy our requirement of consistency. Finally, the conclusions of proposition 4.2 follow immediately from this characterization and the results obtained in Section 3.

Let us first derive the optimality conditions associated to the problem. With this new specification (associated to the new state dynamics $(3,4)$ ), the principle of the maximum can be used. ${ }^{17}$ The associated Hamiltonian is (using (3) and (4)):

$$
H=\beta^{t} \log c_{1, t}+\pi_{t}\left[-a_{2} x_{t}-b_{2} y_{t}-c_{1, t}\right]+\lambda_{t}\left[c_{1, t}-y_{t}\right],
$$

where $\lambda_{t}$ and $\pi_{t}$ denote the adjoint variables related to the two constraints of the present optimization problem. For any period $t$ the first order conditions are:

$$
\begin{gathered}
\frac{\partial H}{\partial c_{1, t}}=\frac{\beta^{t}}{c_{1, t}}-\pi_{t}+\lambda_{t}=0, \\
\pi_{t}-\pi_{t-1}=-\frac{\partial H}{\partial x_{t}}=a_{2} \pi_{t}, \\
\lambda_{t}-\lambda_{t-1}=-\frac{\partial H}{\partial y_{t}}=b_{2} \pi_{t}+\lambda_{t} .
\end{gathered}
$$

From conditions (6) we obtain:

$$
c_{1, t}=\frac{\beta^{t}}{\pi_{t}-\lambda_{t}} ; \quad \pi_{t}=\frac{\pi_{0}}{\left(1-a_{2}\right)^{t}} ; \quad \lambda_{t}=-b_{2} \pi_{t+1} .
$$

Using these expressions we can rewrite $\pi_{t}-\lambda_{t}$ as

$$
\pi_{t}-\lambda_{t}=\frac{\pi_{0}}{\left(1-a_{2}\right)^{t}}+\frac{b_{2} \pi_{0}}{\left(1-a_{2}\right)^{t+1}}=\frac{\pi_{0}}{\left(1-a_{2}\right)^{t}} \frac{1+b_{2}-a_{2}}{1-a_{2}} .
$$

Thus, we obtain a final expression of the optimal consumption plan:

$$
c_{1, t}=\left[\beta\left(1-a_{2}\right)\right]^{t} \frac{1-a_{2}}{\pi_{0}\left(1+b_{2}-a_{2}\right)} .
$$

\footnotetext{
${ }^{17}$ It should be noted that the use of conjectures enables us to move from a dynamic game to a problem of optimal control. This is why we can use the principle of the maximum here.
} 
Using this expression for $t=0$, we can deduce an expression of the initial parameter $\pi_{0}$ as:

$$
\pi_{0}=\frac{1-a_{2}}{c_{1,0}\left(1+b_{2}-a_{2}\right)} .
$$

Now we can rewrite the expression of the state dynamics. Let us denote

$$
A=\frac{b_{2}+\beta\left(1-a_{2}\right)}{\beta\left(1+b_{2}-a_{2}\right) \pi_{0}}
$$

now we can obtain that

$$
\begin{aligned}
& b_{2} c_{1, t-1}+c_{1, t}= \\
& b_{2}\left(\beta\left(1-a_{2}\right)\right)^{t-1} \frac{1-a_{2}}{\pi_{0}\left(1+b_{2}-a_{2}\right)}+\left(\beta\left(1-a_{2}\right)\right)^{t} \frac{1-a_{2}}{\pi_{0}\left(1+b_{2}-a_{2}\right)}= \\
& \left(\beta\left(1-a_{2}\right)\right)^{t} A .
\end{aligned}
$$

Thus, the state dynamics can be rewritten as:

$$
x_{t+1}=\left(1-a_{2}\right) x_{t}-b_{2} c_{1, t-1}-c_{1, t}=\left(1-a_{2}\right) x_{t}-\left[\beta\left(1-a_{2}\right)\right]^{t} A ;
$$

or

$$
x_{t}=\left(1-a_{2}\right)^{t} x_{0}-A\left(1-a_{2}\right)^{t-1} \sum_{i=0}^{t-1} \beta^{i}=\left(1-a_{2}\right)^{t} x_{0}-A\left(1-a_{2}\right)^{t-1} \frac{1-\beta^{t}}{1-\beta} .
$$

Up to now there are several potential solutions to the problem (that is, several possible pairs of parameters $\left.\left(a_{2}, b_{2}\right)\right)$.

Now we solve for the feedback consistent solutions. We consider symmetric solutions because we deal with a symmetric problem. Basically this amounts to assuming $c_{1,0}=c_{2,0}=c_{0}$, that is, the initial level of consumption is the same for both agents. Since the problem is symmetric, we focus on a symmetric solution too. This implies $c_{1, t}=c_{2, t}=c_{t}$ for any period $t$, hence $a_{1}=a_{2}=a$, and $b_{1}=b_{2}=b$.

Now we come back to feedback consistency. As said previously, the state of the process is given (for any period $t$ ) by $\left(x_{t}, c_{t-1}\right)$. Feedback consistency requires that an agent's consumption strategy as conjectured by the other agent corresponds to his actual optimal policy. Formally, we must have:

$$
a x_{t}+b c_{t-1}=c_{t} \text {. }
$$


Thus, to obtain the feedback consistent solutions, one can rewrite condition (11) by using the expressions of $c_{t}$ and $x_{t}$ obtained in (7) and (10), and then obtain the corresponding values of coefficients $a$ and $b$.

Rewriting condition (11), we have:

$$
\begin{gathered}
c_{t}=[\beta(1-a)]^{t} \frac{1-a}{\pi_{0}(1+b-a)}= \\
a\left[(1-a)^{t} x_{0}-A(1-a)^{t-1} \frac{1-\beta^{t}}{1-\beta}\right]+b[\beta(1-a)]^{t-1} \frac{1-a}{\pi_{0}(1+b-a)} .
\end{gathered}
$$

Using the expression of $A$ given by (9) and rewriting, we obtain:

$$
\begin{aligned}
c_{t}= & {[\beta(1-a)]^{t} \frac{1-a}{\pi_{0}(1+b-a)}=(1-a)^{t}\left[a x_{0}-\frac{a}{(1-a)(1-\beta)} \frac{b+\beta(1-a)}{\beta \pi_{0}(1+b-a)}\right] } \\
& +[\beta(1-a)]^{t}\left\{\frac{a[b+\beta(1-a)]}{\beta \pi_{0}(1-a)(1-\beta)(1+b-a)}+\frac{b(1-a)}{\beta \pi_{0}(1-a)(1+b-a)}\right\} .
\end{aligned}
$$

Now feedback consistency is equivalent to looking for values of coefficients $a$ and $b$ such that:

$$
a x_{0}-\frac{a}{(1-a)(1-\beta)} \frac{b+\beta(1-a)}{\beta \pi_{0}(1+b-a)}=0
$$

and

$$
\frac{a[b+\beta(1-a)]}{\beta \pi_{0}(1-a)(1-\beta)(1+b-a)}+\frac{b(1-a)}{\beta \pi_{0}(1-a)(1+b-a)}=\frac{1-a}{\pi_{0}(1+b-a)} .
$$

It is easily checked that conditions (12) and (13) yield the following solutions:

$a=\frac{x_{0} \beta-x_{0}+2 c_{0}}{x_{0} \beta}, b=\frac{\left(x_{0}-2 c_{0}\right)\left(c_{0} \beta-2 c_{0}-x_{0} \beta+x_{0}\right)}{\beta c_{0} x_{0}}=\frac{x_{0}-2 c_{0}}{x_{0}}-\frac{x_{0}-2 c_{0}}{c_{0}} a$.

We now prove proposition 4.2. The proof is straigthforward because by construction

$$
c_{t}^{f c}=[\beta(1-a)]^{t} c_{0}, \quad x_{t}^{f c}=\frac{c_{t}^{f c}-b c_{t-1}^{f c}}{a} .
$$

The optimality of corresponding policies results from the signs of $a$ and $b$, which in turn follow from (14) and (5). The comparisons follow immediately from the forms of optimal policies derived in sections 3.1 and 3.2. This concludes the proof. 


\section{References}

[1] Benhabib, J. and R. Radner (1992), The joint exploitation of a productive asset: A game-theoretic approach, Economic Theory 2, 155-190.

[2] Bischi, G.-I., Kopel, M. and F. Szidarowszky (2005), Expectation-stock dynamics in multi-agent fisheries, Annals of Operations Research 137, 299-329.

[3] Dixon, H. and E. Somma (2003), The evolution of consistent conjectures, Journal of Economic Behavior and Organization 51, 523-536.

[4] Dutta, P. and R. Sundaram (1993), The tragedy of the commons?, Economic Theory 3, 413-426.

[5] Fershtman, C. and M. Kamien (1985), Conjectural equilibrium and strategy spaces in differential games, Optimal Control Theory and Economic Analysis 2, 569-579.

[6] Figuières, C., Jean-Marie, A., Quérou, N. and M. Tidball (2004), Theory of Conjectural Variations, in Monograph series in Mathematical Economics and Game Theory, World Scientific Publishing.

[7] Fischer, R. and L. Mirman (1992), Strategic dynamic interactions: Fish wars, Journal of Economics Dynamics and Control 16, 267-287.

[8] Fischer, R. and L. Mirman (1996), The complete fish wars: biological and dynamic interactions, Journal of Environmental Economics and Management 30, 34-42.

[9] J. Friedman (1977), Oligopoly and the theory of games, North-Holland, Amsterdam.

[10] Friedman, J. and C. Mezzetti (2002), Bounded rationality, dynamic oligopoly, and conjectural variations, Journal of Economic Behavior and Organization 49, 287-306.

[11] Houba, H., Sneek, K. and F. Vardy (2000), Can negotiations prevent fish wars?, Journal of Economic Dynamics and Control 24, 1265-1280.

[12] Jean-Marie, A. and M. Tidball (2005), Consistent conjectures, equilibria and dynamic games, in Dynamic Games: Theory and Applications. Editors: A. Haurie \& G. Zaccour, Springer, 93-109. 
[13] Jean Marie, A. and M. Tidball (2006), Adapting behaviors through a learning process, Journal of Economic Behavior and Organization 60, 399-422.

[14] Kalashnikov, V., Kemfert, C. and V. Kalashnikov (2009), Conjectural variations equilibrium in a mixed duopoly, European Journal of Operational Research 192, 717-729.

[15] Koulovatianos, C., Mirman, L. and M. Santugini (2009), Optimal growth and uncertainty: Learning, Journal of Economic Theory 144, 280-295.

[16] J. Laitner (1980), Rational duopoly equilibria, Quarterly Journal of Economics , 641-662.

[17] Levhari, D. and L. Mirman (1980), The Great Fish War: an example using a dynamic Cournot-Nash solution, Bell Journal of Economics 11, 322-334.

[18] T. Lindh (1992), The inconsistency of consistent conjectures. Coming back to Cournot, Journal of Economic Behavior and Organization 18, 69-90.

[19] Lopez de Haro, S., Sanchez Martn, P., de la Hoz Ardiz, J.E. and J. Fernandez Caro (2007), Estimating conjectural variations for electricity market models, European Journal of Operational Research 181, 13221338 .

[20] R. McKitrick (1999), A Cournot mechanism for pollution control under asymmetric information, Environmental and Resource Economics 14, 353-363.

[21] Muller, W. and H.-T. Normann (2005), Conjectural variations and evolutionary stability: a rationale for consistency, Journal of Institutional and Theoretical Economics 161, 491-502.

[22] A. Possajennikov, The evolutionary stability of constant consistent conjectures, Journal of Economic Behavior and Organization 2009, forthcoming.

[23] Quérou, N. and M. Tidball (2010), Incomplete information, learning, and natural resource management, European Journal of Operational Research 204, 630-638. 
[24] M. Slade (1995), Empirical Games: the oligopoly case, Revue Canadienne D'Economique 28, 368-402. 